\title{
gु \\ X-ray cross-correlation analysis and local symmetries of disordered systems: General theory
}

\author{
M. Altarelli, ${ }^{1,2}$ R. P. Kurta, ${ }^{2}$ and I. A. Vartanyants ${ }^{2, *}$ \\ ${ }^{1}$ European X-ray Free-Electron Laser Facility, Notkestraße 85, D-22607 Hamburg, Germany \\ ${ }^{2}$ Deutsches Elektronen-Synchrotron (DESY), Notkestraße 85, D-22607 Hamburg, Germany
}

(Received 13 June 2010; published 20 September 2010)

\begin{abstract}
In a recent paper [P. Wochner et al., Proc. Natl. Acad. Sci. U.S.A. 106, 11511 (2009)] x-ray scattering intensity correlations around a ring, in the speckle diffraction pattern of a colloidal glass, were shown to display a remarkable $\sim \cos (n \varphi)$ dependence on the angular coordinate $\varphi$ around the ring, with integer index $n$ depending on the magnitude of the scattering wave vector. With an analytical derivation that preserves full generality in the Fraunhofer diffraction limit, we clarify the relationship between this result and previous x-ray studies of bond-orientation order, and provide a sound basis to the statement that the angular intensity correlations deliver information on local bond arrangements in a disordered (or partially ordered) system. We present a detailed analysis of the angular cross-correlation function and show its applicability for studies of a wide range of structural properties of disordered systems, from local structure to spatial correlations between distant structural elements.
\end{abstract}

DOI: 10.1103/PhysRevB.82.104207

PACS number(s): 61.05.cp, 61.43.Dq, 61.43.Fs

\section{INTRODUCTION}

In a recent experiment ${ }^{1}$ by Wochner et al., partially coherent $x$ rays with a wavelength of $0.154 \mathrm{~nm}$ were used to investigate a colloidal glass composed of polymethyl methacrylate (PMMA) spheres of $117 \mathrm{~nm}$ radius, with particle suspensions of concentration exceeding the glass formation value. The scattering pattern on a two-dimensional (2D) detector has the concentric ring structure characteristic of disordered systems, and the speckled appearance results from the partial coherence of the undulator $\mathrm{x}$ rays at the European Synchrotron Radiation Facility. The authors of Ref. 1 have introduced the four-point angular cross-correlation function $(\mathrm{CCF}) C_{q}(\Delta)$ defined as

$$
C_{q}(\Delta)=\frac{\langle I(q, \varphi) I(q, \varphi+\Delta)\rangle_{\varphi}-\langle I(q, \varphi)\rangle_{\varphi}^{2}}{\langle I(q, \varphi)\rangle_{\varphi}^{2}},
$$

where $I(q, \varphi)$ is the scattered intensity, $q$ is the magnitude of the scattering vector $\mathbf{q}, \varphi$ is an angular coordinate around a diffraction ring of radius $q$, and

$$
\langle F(\varphi)\rangle_{\varphi}=\frac{1}{2 \pi} \int_{0}^{2 \pi} F(\varphi) d \varphi
$$

denotes an angular average around the ring. ${ }^{2}$

The remarkable observation by Wochner et al. ${ }^{1}$ is that, at least for some $q$ values, $C_{q}(\Delta)$ is well approximated by a cosine function of an integer multiple of the angle $\Delta$, i.e., $C_{q}(\Delta) \sim \cos (n \Delta)$; different values of $n$ are observed for different $q$. In particular, the experiment on PMMA spheres, for $q \simeq 0.04 \mathrm{~nm}^{-1}$, showed a very pronounced cosine behavior with $n=5$. The authors recovered similar behavior from a numerical simulation, assuming that icosahedral clusters are preferentially formed locally, and computing the scattering intensity and its correlations for a cubic lattice of 8000 such clusters, with random rotational orientation.

In the following, we derive analytical expressions for the Fourier series expansion of the correlation function in the $0 \leq \Delta<2 \pi$ interval, from which the cosinelike behavior of the angular correlation function is related to the arrangement and orientation of bond angles and interatomic distances in the system in a completely general way. We limit ourselves to the Fraunhofer diffraction case here and leave the discussion of the Fresnel diffraction to a forthcoming publication. One interesting aspect of this phenomenon is that it is essentially two-dimensional in character; in a disordered threedimensional (3D) system, it appears that among randomly oriented local bond arrangements, the largest effects are expected from local bond arrangements where $n$-fold symmetry axes are (at least approximately) lined up with the direction of the incident $x$ rays. This leads us to investigate the relationship to pure 2D systems. In fact, extremely marked effects, unveiled by previous x-ray studies, performed with $\mathrm{x}$-ray beams with a limited degree of coherence, of bondorientational order in liquid crystals $^{3-5}$ (especially hexatic ones) and dynamical light scattering experiments ${ }^{6}$ performed with coherent laser beams on ordered 2D colloid systems, are strongly related to the recent results of Wochner et al. Our aim is to provide a sound basis to the statement that the angular intensity correlations deliver information on local bond arrangements in a disordered (or partially ordered) system. In the dilute limit (where local entities containing specific bond angles are separated by distances much larger than the bond lengths) the angular correlation function can be explicitly related to a bond-orientational order parameter, which generalizes the order parameter proposed for hexatic liquid crystals ${ }^{7}$ by Bruinsma and Nelson. ${ }^{8}$

In this paper, we give a general theoretical treatment of the problem of the x-ray cross-correlation analysis in a partially disordered system. In the forthcoming paper, we will present the results of various simulations that demonstrate the general findings presented here. This paper is organized in the following way. In the next section a Fourier series analysis of the intensity angular correlations is presented. In the third section a general theoretical treatment of the CCF is given and the expressions for the Fourier coefficients of the CCFs are derived for the case of a kinematical x-ray scattering. In the fourth section the contribution of different terms 
to the CCF is analyzed. Special treatment is given to dilute and close-packed systems. In the fifth section we consider correlations in 3D systems, when the effect of the Ewald sphere curvature becomes important. Conditions, at which the angular CCF shows $\cos (n \Delta)$ behavior with odd $n$ numbers will be analyzed. The paper is completed by the conclusions and outlook section.

\section{FOURIER SERIES ANALYSIS OF THE INTENSITY ANGULAR CORRELATIONS}

We generalize the CCF defined in Eq. (1) by introducing the intensity correlations at two different magnitudes of the momentum transfer vectors ${ }^{9} q_{1}$ and $q_{2}$,

$$
C_{q_{1}, q_{2}}(\Delta)=\frac{\left\langle I\left(q_{1}, \varphi\right) I\left(q_{2}, \varphi+\Delta\right)\right\rangle_{\varphi}-\left\langle I\left(q_{1}, \varphi\right)\right\rangle_{\varphi} \cdot\left\langle I\left(q_{2}, \varphi\right)\right\rangle_{\varphi}}{\left\langle I\left(q_{1}, \varphi\right)\right\rangle_{\varphi} \cdot\left\langle I\left(q_{2}, \varphi\right)\right\rangle_{\varphi}}
$$

where the averaging over the angle $\varphi$ is defined in Eq. (2). In the next section we will show, that the magnitudes of the scattering vectors $q_{1}$ and $q_{2}$ are, in fact, the values of the perpendicular components of the $3 \mathrm{D}$ scattering vectors $\mathbf{q}_{1}$ and $\mathbf{q}_{2}$. One can readily see that the CCF [Eq. (3)] can be rewritten in a slightly different form

$$
\begin{aligned}
C_{q_{1}, q_{2}}(\Delta) & \\
= & \frac{\left\langle\left(I\left(q_{1}, \varphi\right)-\left\langle I\left(q_{1}, \varphi\right)\right\rangle_{\varphi}\right) \cdot\left(I\left(q_{2}, \varphi+\Delta\right)-\left\langle I\left(q_{2}, \varphi\right)\right\rangle_{\varphi}\right)\right\rangle_{\varphi}}{\left\langle I\left(q_{1}, \varphi\right)\right\rangle_{\varphi} \cdot\left\langle I\left(q_{2}, \varphi\right)\right\rangle_{\varphi}},
\end{aligned}
$$

which shows that we are dealing with the angular correlation function of a normalized deviation of the intensity on the diffraction ring. Let us define this quantity for each value of the momentum transfer vector $q_{j}$ as

$$
D_{I}\left(q_{j}, \varphi\right)=\frac{I\left(q_{j}, \varphi\right)-\left\langle I\left(q_{j}, \varphi\right)\right\rangle_{\varphi}}{\left\langle I\left(q_{j}, \varphi\right)\right\rangle_{\varphi}}, \quad j=1,2
$$

and notice that this function has obviously a vanishing angular average. The measured correlation function [Eq. (3)] can therefore be written as

$$
C_{q_{1}, q_{2}}(\Delta)=\left\langle D_{I}\left(q_{1}, \varphi\right) D_{I}\left(q_{2}, \varphi+\Delta\right)\right\rangle_{\varphi} .
$$

In order to understand what periodicity or symmetry this function of $\Delta$ may display, let us now proceed to its expansion into a Fourier series in the $(0,2 \pi)$ interval

$$
\begin{gathered}
C_{q_{1}, q_{2}}(\Delta)=\sum_{n=-\infty}^{\infty} C_{q_{1}, q_{2}}^{n} e^{i n \Delta}, \\
C_{q_{1}, q_{2}}^{n}=\frac{1}{2 \pi} \int_{0}^{2 \pi} C_{q_{1}, q_{2}}(\Delta) e^{-i n \Delta} d \Delta .
\end{gathered}
$$

Here $C_{q_{1}, q_{2}}^{n}$ is the $n$th coefficient in the Fourier series expansion of $C_{q_{1}, q_{2}}(\Delta)$. Substituting now expression (6) into Eq. (7b) and following the usual arguments for the Fourier transforms of convolutions we get $^{10}$

$$
C_{q_{1}, q_{2}}^{n}=D_{I}^{n *}\left(q_{1}\right) D_{I}^{n}\left(q_{2}\right),
$$

where $D_{I}^{n}\left(q_{j}\right)$ are the Fourier coefficients of a normalized deviation of the intensity. One can see that in order to calculate the Fourier coefficients of $C_{q_{1}, q_{2}}(\Delta)$, one may first calculate those of $D_{I}\left(q_{j}, \varphi\right)$, i.e., $D_{I}^{n}\left(q_{j}\right)$ and then take a product according to Eq. (8). Note that the definition [Eq. (5)] of the normalized deviation $D_{I}\left(q_{j}, \varphi\right)$ implies that $D_{I}^{0}\left(q_{j}\right)=0$. Since scattered intensities are always real quantities, it is also easy to show that $D_{I}^{-n}\left(q_{j}\right)=D_{I}^{n *}\left(q_{j}\right)$ and, therefore, $C_{q_{1}, q_{2}}^{-n}=C_{q_{1}, q_{2}}^{n *}$. According to these symmetry conditions Eq. (7a) can be represented in the following form:

$$
C_{q_{1}, q_{2}}(\Delta)=2 \sum_{n=1}^{\infty} \operatorname{Re}\left[C_{q_{1}, q_{2}}^{n} e^{i n \Delta}\right]=2 \sum_{n=1}^{\infty}\left|C_{q_{1}, q_{2}}^{n}\right| \cdot \cos \left(n \Delta+\gamma_{n}\right),
$$

where $\gamma_{n}=\arg \left(C_{q_{1}, q_{2}}^{n}\right)$ and the summation is performed over the positive integer numbers $n$.

In the particular case, when $q_{1}=q_{2}=q$, Eqs. (8) and (9) reduce to

$$
\begin{gathered}
C_{q}(\Delta)=2 \sum_{n=1}^{\infty} C_{q}^{n} \cos (n \Delta), \\
C_{q}^{n}=\left|D_{I}^{n}(q)\right|^{2}, \quad C_{q}^{n} \geq 0 .
\end{gathered}
$$

The general analysis of CCFs presented in this section and particularly Eqs. (10a) and (10b) explain a single cosine behavior of CCF calculated from experimental data in Ref. 1. Clearly, a strong single cosine dependence of $C_{q}(\Delta)$ can be observed only for those values of $q$, at which one of the Fourier coefficients $C_{q}^{n}$ significantly dominates over all others. In the following sections we will show how such coefficients can be related to the structure and symmetry of the system.

It is to be noted that Eqs. (10a) and (10b) also imply that the analysis of the CCF [Eq. (1)] investigated by Wochner et $a l .{ }^{1}$ does not really contain additional information with respect to the Fourier analysis of the $\varphi$ dependence of the intensity. Examples ${ }^{3-5}$ of analysis of the periodicity in the angular dependence of the intensity can be found in studies of hexatic liquid crystal phases, ${ }^{7}$ performed with $\mathrm{x}$-ray beams with the limited degree of coherence. In the next section, we present detailed derivations of the $\mathrm{CCF}$, based on the kinematical x-ray scattering theory.

\section{GENERAL THEORETICAL TREATMENT OF THE CROSS-CORRELATION FUNCTION}

We start our discussion with a simple scattering geometry depicted in Fig. 1. A coherent x-ray beam scatters from a disordered sample and creates a speckle pattern on the detector in the far-field regime. As a general model system we assume a 3D sample consisting of identical 3D local structures (LSs) of arbitrary shape, random orientation, and position in 3D space (Fig. 2). Such a model includes a variety of systems, i.e., clusters or molecules in the gas phase, LSs 


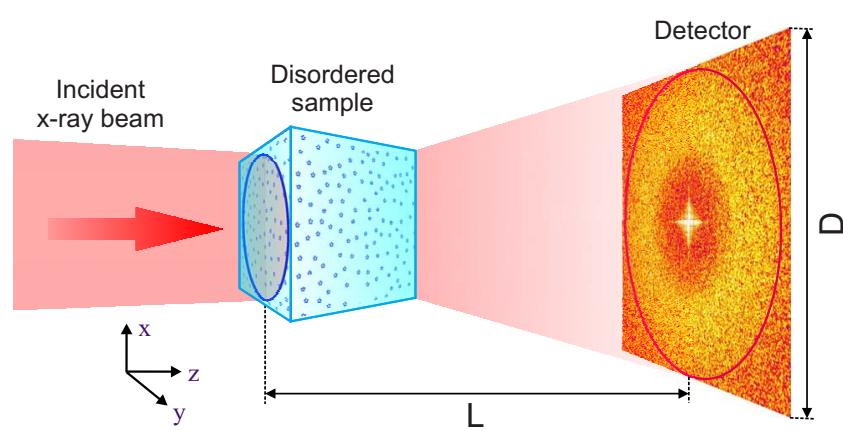

FIG. 1. (Color online) Geometry of the diffraction experiment. A coherent x-ray beam illuminates a disordered sample and produces a speckle diffraction pattern on a detector. The speckle features are defined by the finite size of the beam or the finite size of the sample and its microscopic configuration. The direction of the incident beam is defined along the $z$ axis of the coordinate system.

formed in colloidal systems (similar to Ref. 1), protein molecules, viruses, or complex biological systems in solution.

The coherent x-ray scattering amplitude $A(\mathbf{q})$ from such a sample can be described in the first Born approximation (or kinematical scattering) as

$$
A(\mathbf{q})=\int \rho(\mathbf{r}) e^{i \mathbf{q} \cdot \mathbf{r}} d \mathbf{r},
$$

where $\rho(\mathbf{r})$ is a total electron density of the system. ${ }^{11}$ For the disordered systems under consideration here this electron density can be written in the following form:

$$
\rho(\mathbf{r})=\sum_{k=1}^{N} \rho_{k}\left(\mathbf{r}-\mathbf{R}_{k}\right)
$$

where $\rho_{k}(\mathbf{r})$ is an electron density of the $k$ th LS at the position $\mathbf{R}_{k}$ (see Fig. 2) and the summation is performed over all $N$ LSs. Substituting Eq. (12) into Eq. (11) we obtain for the total scattered amplitude

$$
A(\mathbf{q})=\sum_{k=1}^{N} e^{i \mathbf{q} \cdot \mathbf{R}_{k}} A_{k}(\mathbf{q}),
$$

where $A_{k}(\mathbf{q})$ is the amplitude scattered by one LS,

$$
A_{k}(\mathbf{q})=\int \rho_{k}(\mathbf{r}) e^{i \mathbf{q} \cdot \mathbf{r}} d \mathbf{r}
$$

and the integration is performed over the volume of each LS. Equations (13) and (14) express a simple fact that under conditions of coherent illumination the total scattering amplitude for each value of the wave vector $\mathbf{q}$ is a coherent sum of the individual amplitudes from each LS modulated with the corresponding phase term $\exp \left(i \mathbf{q} \mathbf{R}_{k}\right)$, depending on the position $\mathbf{R}_{k}$ of each LS.

Using Eq. (13), we can write the intensity scattered at the momentum transfer value $\mathbf{q}$ as

$$
I(\mathbf{q})=\sum_{k_{1}, k_{2}=1}^{N} e^{i \mathbf{q} \cdot\left(\mathbf{R}_{k_{2}}-\mathbf{R}_{k_{1}}\right)} A_{k_{1}}^{*}(\mathbf{q}) A_{k_{2}}(\mathbf{q})
$$

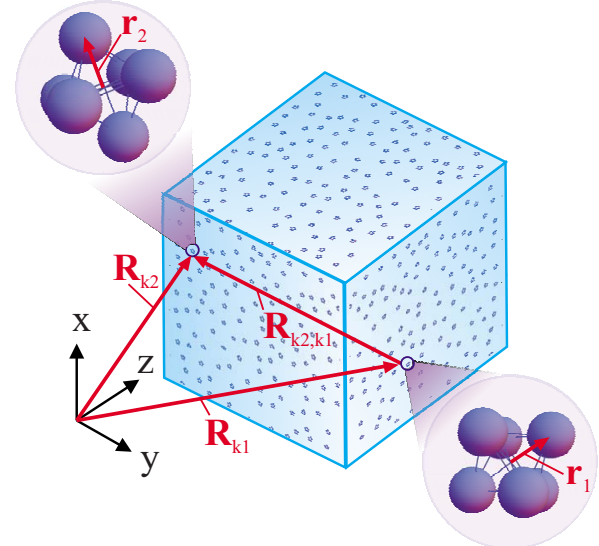

FIG. 2. (Color online) Disordered sample consisting of clusters randomly oriented and distributed in $3 \mathrm{D}$ space. The radius vector $\mathbf{R}_{k_{2}, k_{1}}=\mathbf{R}_{k_{2}}-\mathbf{R}_{k_{1}}$ connects the centers $\mathbf{R}_{k_{1}}$ and $\mathbf{R}_{k_{2}}$ of two different clusters $k_{1}$ and $k_{2}$, the vectors $\mathbf{r}_{1}$ and $\mathbf{r}_{2}$ define the positions of particles inside each cluster with the origin of a local coordinate system in each cluster $k_{i}$ positioned at its center.

$$
\begin{aligned}
= & \sum_{k_{1}, k_{2}=1}^{N} e^{i \mathbf{q} \cdot\left(\mathbf{R}_{k_{2}}-\mathbf{R}_{k_{1}}\right)} \iint \rho_{k_{1}}^{*}\left(\mathbf{r}_{1}\right) \rho_{k_{2}}\left(\mathbf{r}_{2}\right) \\
& \times e^{i \mathbf{q} \cdot\left(\mathbf{r}_{2}-\mathbf{r}_{1}\right)} d \mathbf{r}_{1} d \mathbf{r}_{2} \\
= & \sum_{k_{1}, k_{2}=1}^{N} \iint \rho_{k_{1}}^{*}\left(\mathbf{r}_{1}\right) \rho_{k_{2}}\left(\mathbf{r}_{2}\right) e^{i \mathbf{q} \cdot \mathbf{R}_{k_{2}, k_{1}}^{21} d \mathbf{r}_{1} d \mathbf{r}_{2} .}
\end{aligned}
$$

Here, the following notation for the radius vectors connecting two particles 1 and 2 in two different clusters $k_{1}$ and $k_{2}$ was used

$$
\mathbf{R}_{k_{2}, k_{1}}^{21}=\mathbf{R}_{k_{2}, k_{1}}+\mathbf{r}_{21},
$$

where $\mathbf{R}_{k_{2}, k_{1}}=\mathbf{R}_{k_{2}}-\mathbf{R}_{k_{1}}$ is the radius vector connecting different local structures, and $\mathbf{r}_{21}=\mathbf{r}_{2}-\mathbf{r}_{1}$ is the radius vector connecting subunits inside LSs (see Fig. 2).

We decompose now the scattering vector $\mathbf{q}=\left(\mathbf{q}^{\perp}, q^{z}\right)$ into two components: $\mathbf{q}^{\perp}$ that is perpendicular and $q^{z}$ that is parallel to the direction of the incident beam (see Fig. 3). We define the perpendicular component of the scattering vector $\mathbf{q}^{\perp}$ in polar coordinates as $\mathbf{q}^{\perp}=\left(q^{\perp}, \varphi\right)$. We also define the perpendicular $\mathbf{R}_{k_{2}, k_{1}}^{\perp 21}=\mathbf{R}_{k_{2}, k_{1}}^{\perp}+\mathbf{r}_{21}^{\perp}$, and the $z$ components $Z_{k_{2}, k_{1}}^{21}=Z_{k_{2}, k_{1}}+z_{21}$ of the radius vectors introduced in Eq. (16) (see Figs. 1 and 2). Using these notations for the vectors we can rewrite Eq. (15) as

$I(\mathbf{q})=\sum_{k_{1}, k_{2}=1}^{N} e^{-i q^{z} \cdot Z_{k_{2}, k_{1}}} \iint \tilde{\rho}_{k_{1}}^{*}\left(\mathbf{r}_{1}^{\perp}, q^{z}\right) \widetilde{\rho}_{k_{2}}\left(\mathbf{r}_{2}^{\perp}, q^{z}\right) e^{i \mathbf{q}^{\perp} \cdot \mathbf{R}_{k_{2}, k_{1}}^{\perp 21} d \mathbf{r}_{1}^{\perp} d \mathbf{r}_{2}^{\perp} .}$

Here we introduced a modified complex valued electron density function, defined as

$$
\tilde{\rho}_{k_{i}}\left(\mathbf{r}_{i}^{\perp}, q^{z}\right)=\int \rho_{k_{i}}\left(\mathbf{r}_{i}^{\perp}, z\right) e^{-i q^{z} z} d z .
$$




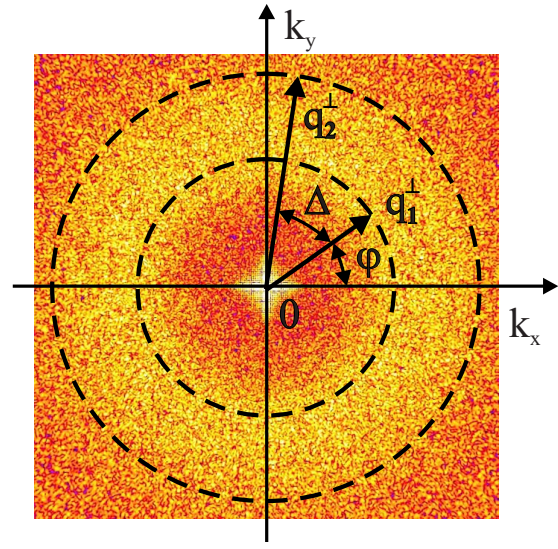

(a)

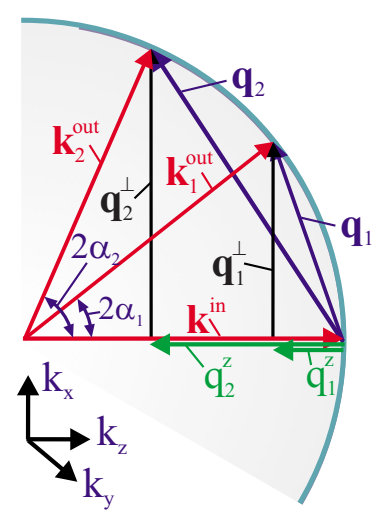

(b)

FIG. 3. (Color online) (a) In the general case, the cross-correlation function can be defined as an angular average over two intensity rings with different magnitudes of the scattering vectors $q_{1}^{\perp} \neq q_{2}^{\perp}$. The perpendicular components of the scattering vectors $\mathbf{q}_{1}^{\perp}$ and $\mathbf{q}_{2}^{\perp}$ are defined in the plane $\left(k_{x}, k_{y}\right)$ in the polar coordinate system as $\mathbf{q}_{1}^{\perp}=\left(q_{1}^{\perp}, \varphi\right)$ and $\mathbf{q}_{2}^{\perp}=\left(q_{2}^{\perp}, \varphi+\Delta\right)$. (b) Scattering geometry in reciprocal space. Here $\mathbf{k}^{\text {in }}$ is the wave vector of the incident beam directed along the $z$ axis, $\mathbf{k}_{1}^{\text {out }}$ and $\mathbf{k}_{2}^{\text {out }}$ are the wave vectors of two scattered waves with the scattering angles $2 \alpha_{1}$ and $2 \alpha_{2}$. The scattering vectors $\mathbf{q}_{1}=\left(\mathbf{q}_{1}^{\perp}, q_{1}^{z}\right)$ and $\mathbf{q}_{2}=\left(\mathbf{q}_{2}^{\perp}, q_{2}^{z}\right)$ are decomposed into two components: $\mathbf{q}_{j}^{\perp}$ that is perpendicular and $q_{j}^{z}$ that is parallel to the direction of the incident beam.

We want to note here that our treatment is quite general and is valid for both cases of wide and small angle scattering. In the first case, the effect of the Ewald sphere curvature [see Fig. 3(b)], which manifests itself by the presence of the exponential factors $e^{-i q^{z} \cdot Z_{k_{2}, k_{1}}}$ and $e^{-i q^{z} z}$ in Eqs. (17) and (18), may become important. This effect could break the scattering symmetry of a diffraction pattern, characteristic for the scattering on a positive valued electron density (Friedel's law) and may reveal additional symmetries that can be still hidden in the small angle scattering case. This wide angle scattering geometry may become important for scattering on atomic systems with local interatomic distances on the order of a few angstroms. In the small angle scattering geometry, with scattering angles $2 \alpha \ll 1$, we have for the values of the scattering vectors: $q \simeq 2 k \alpha\left(1-\alpha^{2} / 6+\cdots\right), q^{\perp} \simeq 2 k \alpha\left(1-2 \alpha^{2} / 3\right.$ $+\cdots)$, and $q^{z} \simeq 2 k \alpha^{2}\left(1-\alpha^{2} / 3+\cdots\right)$. It is well seen from these expressions that the $q^{z}$ component of the momentum transfer vector is proportional to the square of the small scattering angle $\alpha$. It means that, in this situation, the $z$ components of the momentum transfer vectors are much smaller than their perpendicular components, i.e., $q^{z} \ll q^{\perp}$ and can be neglected. In this limit we have a simplified expression for the intensity [Eq. (17)] that does not depend on the $z$ component of the scattering vector $q^{z}$. For a real valued electron density $\rho_{k_{i}}\left(\mathbf{r}_{i}\right)$ the modified electron density function [Eq. (18)] reduces to a real valued projected electron density of a LS

$$
\widetilde{\rho}_{k_{i}}\left(\mathbf{r}_{i}^{\perp}\right)=\int \rho_{k_{i}}\left(\mathbf{r}_{i}^{\perp}, z\right) d z .
$$

This case of a small angle scattering is typical for scattering on colloidal samples with a typical distance between colloidal particles of few hundred nanometers as in Ref. 1.

According to Eq. (8), the Fourier coefficients of the CCF are determined by the Fourier coefficients of the normalized deviation $D_{I}^{n}\left(q_{j}\right)$. Direct calculations (see Appendix A for details) give for $D_{I}^{n}\left(q_{j}\right)$,

$$
D_{I}^{n}\left(q_{j}\right)=I^{n}\left(q_{j}^{\perp}, q_{j}^{z}\right) / I^{0}\left(q_{j}^{\perp}, q_{j}^{z}\right), \quad n \neq 0,
$$

where the Fourier coefficients of the intensity $I^{n}\left(q_{j}^{\perp}, q_{j}^{z}\right)$ are

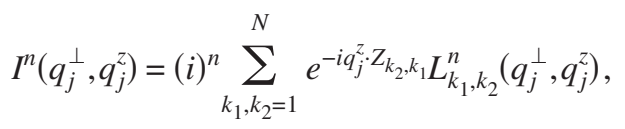

$$
L_{k_{1}, k_{2}}^{n}\left(q_{j}^{\perp}, q_{j}^{z}\right)=\iint d \mathbf{r}_{1}^{\perp} d \mathbf{r}_{2}^{\perp} \widetilde{\boldsymbol{\rho}}_{k_{1}}^{*}\left(\mathbf{r}_{1}^{\perp}, q_{j}^{z}\right) \widetilde{\rho}_{k_{2}}\left(\mathbf{r}_{2}^{\perp}, q_{j}^{z}\right) J_{n}\left(q_{j}^{\perp}\left|\mathbf{R}_{k_{2}, k_{1}}^{\perp 21}\right|\right) e^{-i n \phi_{\mathbf{R}_{k_{2}, k_{1}}^{\perp 21}}}
$$

Here $J_{n}(\rho)$ is the Bessel function of the first kind of integer order $n$, and $\phi_{\mathbf{R}_{k_{2}, k_{1}}^{\perp 21}}$ is the azimuthal angle of the perpendicular component of the radius vector $\mathbf{R}_{k_{2}, k_{1}}^{21}$ defined in Eq. (16) (see Fig. 2).

From the derived expressions we can draw the following important conclusions. According to Eqs. (7a), (7b), (8),
(20), (21a), and (21b), the initial four-point correlation function $C_{q_{1}, q_{2}}(\Delta)$ can be represented by its Fourier series expansion, where each Fourier coefficient is defined by a product of two two-point correlation functions of the form [Eqs. (21a) and (21b)], corresponding to two different momentum transfer vectors $\mathbf{q}_{1}$ and $\mathbf{q}_{2}$. The magnitude of the $n$th coeffi- 
cient is defined by the Fourier coefficients $I^{n}\left(q_{j}^{\perp}, q_{j}^{z}\right)$ in Eq. (21a), which depend through $L_{k_{1}, k_{2}}^{n}\left(q_{j}^{\perp}, q_{j}^{z}\right)$ on the internal symmetry of the LSs as well as on the medium range order of these LSs in the disordered system. We will discuss the structure of these Fourier coefficients for certain scattering geometries in more detail in the next sections.

\section{CCF DECOMPOSITION: LOCAL STRUCTURE AND INTERPARTICLE SPATIAL CORRELATIONS}

In this section we consider more closely the contribution of different terms in the expansion [Eq. (21a)] to the Fourier coefficients $C_{q_{1}, q_{2}}^{n}$. We consider here a particular case of a $2 \mathrm{D}$ system in a small angle scattering geometry $(2 \alpha \ll 1)$, when we can neglect the $z$ components of the scattering vectors $q_{1}^{z}$ and $q_{2}^{z}$. In this case, the modified electron density ${ }^{12} \widetilde{\rho}_{k_{i}}\left(\mathbf{r}_{i}\right)$ is defined by Eq. (19). The sum in expression (21a) for the Fourier coefficients of intensity $I^{n}\left(q_{j}\right)$ can be split into two parts,

$$
\begin{aligned}
I^{n}\left(q_{j}\right) & =(i)^{n} \sum_{k_{1}, k_{2}=1}^{N} L_{k_{1}, k_{2}}^{n}\left(q_{j}\right) \\
& =(i)^{n}\left[\sum_{k_{1}=k_{2}=k}^{N} L_{k}^{n}\left(q_{j}\right)+\sum_{k_{1} \neq k_{2}}^{N} L_{k_{1}, k_{2}}^{n}\left(q_{j}\right)\right],
\end{aligned}
$$

where the first sum corresponds to the terms with $k_{1}=k_{2}=k$ and the last one to the terms with $k_{1} \neq k_{2}$.

\section{A. Dilute systems}

It can be shown (see Appendix B) that for dilute systems, when the average distance $D$ between the clusters is much larger than the size $d$ of a single cluster, the contribution of the second sum in Eq. (22) can become much smaller than that of the first one. In this situation the main contribution to the Fourier coefficients of CCFs will be determined by the first sum in Eq. (22), which we consider in detail below. For the first term in Eq. (22) $k_{1}=k_{2}=k$ and, therefore, $\mathbf{R}_{k_{2}, k_{1}}=0$ and $\mathbf{R}_{k_{2}, k_{1}}^{21}=\mathbf{r}_{21}$. We then have an especially simple expression for the integral $L_{k}^{n}\left(q_{j}\right)$ in Eq. (22)

$$
L_{k}^{n}\left(q_{j}\right)=\iint d \mathbf{r}_{1} d \mathbf{r}_{2} \widetilde{\rho}_{k}\left(\mathbf{r}_{1}\right) \widetilde{\rho}_{k}\left(\mathbf{r}_{2}\right) J_{n}\left(q_{j}\left|\mathbf{r}_{21}\right|\right) e^{-i n \phi_{\mathbf{r}_{21}}}
$$

Here $\phi_{\mathbf{r}_{21}}$ is the azimutal angle of the radius vector $\mathbf{r}_{21}$ connecting two particles in the same LS. If all LSs have the same internal structure but are oriented and located in space randomly, the phase $\phi_{\mathbf{r}_{21}}$ in the exponent of Eq. (23) can be defined as

$$
\phi_{\mathbf{r}_{21}}=\phi_{k}+\phi_{\mathbf{r}_{21}}^{0},
$$

where $\phi_{k}$ is the rotation angle of the $k$ th LS with respect to the fixed angular orientation $\phi_{\mathbf{r}_{21}}^{0}$ of the LS in the origin of the coordinate system. In this case, for each LS the integral [Eq. (23)] can be expressed in the following form:

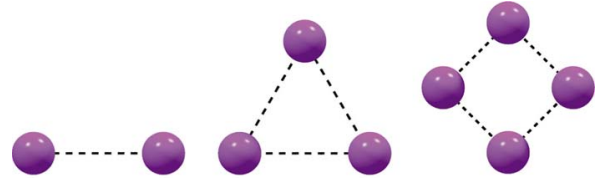

(a)

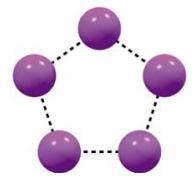

(d) (b) (c)
FIG. 4. (Color online) 2D particles with simple geometrical shapes exhibiting different rotational symmetries (rotational axes are perpendicular to the plane of the figure): (a) twofold, (b) threefold, (c) fourfold, (d) fivefold, and (e) sixfold.

$$
L_{k}^{n}\left(q_{j}\right)=e^{-i n \phi_{k}} L^{n}\left(q_{j}\right)
$$

Here the contribution of each LS $k$ is determined by its rotation angle $\phi_{k}$ in the phase and the integral $L^{n}\left(q_{j}\right)$ is the same for all LSs

$$
L^{n}\left(q_{j}\right)=\iint d \mathbf{r}_{1} d \mathbf{r}_{2} \widetilde{\rho}\left(\mathbf{r}_{1}\right) \widetilde{\rho}\left(\mathbf{r}_{2}\right) J_{n}\left(q_{j}\left|\mathbf{r}_{21}\right|\right) e^{-i n \phi_{\mathbf{r}_{21}}^{0}} .
$$

According to the structure of the integral $L^{n}\left(q_{j}\right)$ its value strongly depends on the symmetry of a LS and determines selection rules for the values $n$ of nonzero Fourier coefficients $C_{q_{1}, q_{2}}^{n}$. These selection rules can be used for the identification of the symmetry of clusters in diluted systems. To demonstrate this, we calculate in Appendix $\mathrm{C}$ the integral $L^{n}\left(q_{j}\right)$ for $2 \mathrm{D}$ clusters with a different rotational symmetry (see Fig. 4). For example, for a cluster with fivefold symmetry $[$ Fig. $4(\mathrm{~d})]$ only $n=10 i,(i=1,2, \ldots)$ will give a nonzero contribution to the Fourier coefficients of CCFs. Note that the Fourier coefficient with $n=5$ is forbidden in this scattering geometry.

The same arguments follow for the zero Fourier component of intensity $I^{0}\left(q_{j}\right)$ that leads us to the following result for dilute systems:

$$
I^{0}\left(q_{j}\right) \approx \sum_{k_{1}=k_{2}=k}^{N} L_{k}^{0}\left(q_{j}\right)=N L^{0}\left(q_{j}\right)
$$

where the integral $L^{0}\left(q_{j}\right)$ does not depend on the index $k$ and is defined by the expression

$$
L^{0}\left(q_{j}\right)=\iint d \mathbf{r}_{1} d \mathbf{r}_{2} \widetilde{\rho}\left(\mathbf{r}_{1}\right) \widetilde{\rho}\left(\mathbf{r}_{2}\right) J_{0}\left(q_{j}\left|\mathbf{r}_{21}\right|\right) .
$$

Taking all of this into account, we have in the limit of dilute systems [neglecting the second term in Eq. (22)] for the Fourier coefficients $C_{q_{1}, q_{2}}^{n}$ of the CCF the following expression: 


$$
\begin{aligned}
C_{q_{1}, q_{2}}^{n}=D_{I}^{n *}\left(q_{1}\right) D_{I}^{n}\left(q_{2}\right) & =\frac{\mathcal{L}^{n}\left(q_{1}, q_{2}\right)}{N^{2}} \sum_{k=1}^{N} \sum_{k^{\prime}=1}^{N} e^{i n\left(\phi_{k}-\phi_{k^{\prime}}\right)} \\
& =\frac{\mathcal{L}^{n}\left(q_{1}, q_{2}\right)}{N^{2}}\left|\sum_{k=1}^{N} e^{i n \phi_{k}}\right|^{2},
\end{aligned}
$$

where

$$
\mathcal{L}^{n}\left(q_{1}, q_{2}\right)=L^{n *}\left(q_{1}\right) L^{n}\left(q_{2}\right) /\left[L^{0}\left(q_{1}\right) L^{0}\left(q_{2}\right)\right] .
$$

The sum in Eq. (29) is well known in statistical optics as a random phasor sum ${ }^{13,14}$ and can be written in the following form: ${ }^{15}$

$$
\frac{1}{N} \sum_{k=1}^{N} e^{i n \phi_{k}}=A e^{i \theta}=\left\langle e^{i n \phi}\right\rangle_{\phi}
$$

that leads to the final result for $C_{q_{1}, q_{2}}^{n}$,

$$
C_{q_{1}, q_{2}}^{n}=\mathcal{L}^{n}\left(q_{1}, q_{2}\right) A^{2}=\mathcal{L}^{n}\left(q_{1}, q_{2}\right)\left|\left\langle e^{i n \phi}\right\rangle_{\phi}\right|^{2} .
$$

In Eq. (31) $A$ and $\theta$ are the amplitude and phase of the random phasor sum and the average $\langle\cdots\rangle_{\phi}$ is performed over all local structure orientations $\phi$ and can be defined as

$$
\left\langle e^{i n \phi}\right\rangle_{\phi}=\int p(\phi) e^{i n \phi} d \phi,
$$

where

$$
p(\phi)=1 / N \sum_{k=1}^{N} \delta\left(\phi-\phi_{k}\right)
$$

is the probability distribution of angular orientations. The average $\left\langle e^{i n \phi}\right\rangle_{\phi}$ is, in fact, a generalization, for $n \neq 6$, of the bond-orientational order parameter, introduced for hexatic liquid crystals ${ }^{7}$ by Bruinsma and Nelson. ${ }^{8}$

We want to emphasize here that in the case of dilute systems we obtained an extremely transparent result. Fourier coefficients of the $\mathrm{CCF} C_{q_{1}, q_{2}}^{n}$ [Eq. (32)] are determined by the product of two factors. The first one, $\mathcal{L}^{n}\left(q_{1}, q_{2}\right)$ [Eq. (30)] depends on the structure and internal symmetry of the LSs and the second one is given by the square amplitude value of the random phasor sum [Eq. (31)] $A^{2}=\left|\left\langle e^{i n \phi}\right\rangle_{\phi}\right|^{2}$ and depends on the concrete realization of the system, i.e., the set of orientations $\phi_{k}$. Its value fluctuates from one set of orientations to another, fluctuations being greater for a smaller number of different orientations $\phi_{k}$. In an experiment performed on a dynamic system the values of the CCF $C_{q_{1}, q_{2}}(\Delta)$ (or Fourier components of it, $C_{q_{1}, q_{2}}^{n}$ ) will also fluctuate in time giving a different result for the same system in measurements performed over a certain time interval. The statistics of these fluctuations is determined by the statistics of the phase distribution $\phi$. Below we will consider a few possible scenarios for this phase distribution for a large number of particles in the illuminated volume.

First, we will discuss two different limits for the possible orientations of LSs in a 2D plane. If all LSs have the same angular orientation, i.e., all $\phi_{k}=\phi_{0}$, then the probability distribution function $p(\phi)$ reduces to a single delta function $p(\phi)=\delta\left(\phi-\phi_{0}\right)$. In this case of a completely oriented system $\left\langle e^{i n \phi}\right\rangle_{\phi}=e^{i n \phi_{0}}$ and $\left|\left\langle e^{i n \phi}\right\rangle\right|^{2}=1$. This means that nonzero values of the Fourier coefficients $C_{q_{1}, q_{2}}^{n}$ [Eq. (32)] will be determined only by the values of the factor $\mathcal{L}^{n}\left(q_{1}, q_{2}\right)$ [Eq. (30)] evaluated for one LS in the system. Clearly, no fluctuations of $C_{q_{1}, q_{2}}^{n}$ will be observed in such a system.

In another limiting case, a uniform distribution of orientations $\phi_{k}$, the statistics of the amplitude $A$ of the random phasor sum [Eq. (31)] is well known, ${ }^{14}$ and can be described by the Rayleigh density function for a large number of different orientations, or phases $\phi_{k}$. We found that the statistics of $A^{2}$, which determines the values of the Fourier coefficients of the CCF [Eq. (32)] for the same uniform distribution of phases $\phi_{k}$, is different and can be described for a large number of different phases by the exponential probability density function $p\left(A^{2}\right)=\lambda e^{-\lambda A^{2}}$, where the rate parameter $\lambda=N$ in our case. This distribution has a mean value $\left\langle A^{2}\right\rangle=1 / \lambda=1 / N$ and a variance $\sigma_{A^{2}}^{2}=1 / \lambda^{2}=1 / N^{2}$. It means that the values of $A^{2}$ and, consequently, of the Fourier coefficients of the CCF $C_{q_{1}, q_{2}}^{n}$ [Eq. (32)] in the case of a dynamic system will fluctuate around the average value $1 / N$ with the standard deviation of these fluctuations given by $\sigma_{A^{2}}=1 / N$. It is clear that in the limit of $N \rightarrow \infty$, the Fourier coefficients of the CCF have vanishing values, ${ }^{16} C_{q_{1}, q_{2}}^{n} \rightarrow 0$. According to these results, for dilute systems with a large number $N$ and a uniform distribution of orientations $\phi_{k}$, it becomes practically impossible to determine the symmetry of the LSs from the analysis of the angular CCF. This is similar to the situation in small angle $\mathrm{x}$-ray scattering, when there is no preferential orientation in the disordered system. If a nonzero Fourier coefficient is observed, it implies either a preferential alignment along a given direction, by a specific physical reason, or, alternatively, that the ensemble of probed LSs is small enough to display pronounced fluctuations from the average uniform distribution of orientations.

In the case of partial ordering, the angular orientations of LSs can be described, for example, by a Gaussian distribution. Such a situation may be realized when a disordered system is in an external field (magnetic, electric, etc.), which drives it toward a more ordered state. In this case the orientational probability distribution is given by

$$
p(\phi)=1 /\left(\sqrt{2 \pi} \sigma_{\phi}\right) \exp \left[-\phi^{2} /\left(2 \sigma_{\phi}^{2}\right)\right],
$$

where $\sigma_{\phi}$ is the standard deviation and we assume a zero mean $\langle\phi\rangle=0$. Unfortunately, in this case of a nonuniform distribution of phases, there is no closed form solution for a probability density function of $A^{2}$ describing its statistics. However, the average value $\left\langle A^{2}\right\rangle$ can be found following the procedure described in Ref. 14 for a large number of phases $\phi_{k}$ :

$$
\left\langle A^{2}\right\rangle=\exp \left(-n^{2} \sigma_{\phi}^{2}\right)(1-1 / N)+1 / N .
$$

Following our previous discussion this means that the values of the Fourier components of the CCF for a dynamic system will fluctuate around this average value. For a broad distribution of phases $\left(\sigma_{\phi} \gg 1\right)$ we obtain for $\left\langle A^{2}\right\rangle$ the same result as in the case of a uniform distribution of orientations discussed above. For a number of particles $N \rightarrow \infty$ we obtain 
from Eq. (36) for the Fourier components of the angular CCF [Eq. (32)] (Ref. 17)

$$
C_{q_{1}, q_{2}}^{n}=\mathcal{L}^{n}\left(q_{1}, q_{2}\right) \exp \left(-n^{2} \sigma^{2}\right) .
$$

We see that in the case of partial ordering in a system with a Gaussian distribution of orientations of LSs, and in the limit of $N \rightarrow \infty$, the number of Fourier coefficients of the $\mathrm{CCF}$ is limited. The strongest contribution to the Fourier coefficients $C_{q_{1}, q_{2}}^{n}$ is given by the lowest values of $n$ and is stronger for more ordered systems (that correspond to lower values of $\sigma$ ).

Here we want also to note the following. In our general treatment of CCFs we assumed a fully coherent incoming beam [see Eq. (11)]. However, it is clear from our discussion of CCFs in dilute systems that the requirements on coherence can be significantly relaxed. In the case of a reduced transverse coherence length, all our results presented here are still valid. The coherence length can even go below the average distance between LSs but, importantly, it should be larger than the size of a LS. The only difference in the observed diffraction pattern will be that instead of a speckle diffraction pattern with high fluctuations of intensity, typical for coherent scattering from disordered systems depicted in Fig. 1, the intensity distribution will be smoother. This is similar to the situation analyzed in Ref. 18, where it was demonstrated by simulations on completely oriented system of particles that when the transverse coherence length of the incoming beam is on the order of the size of a single particle, the resulting diffraction pattern from the ensemble of particles will be similar to the one produced by a single particle. Though a large transverse coherence length is less important for the analysis of dilute systems, it can be still important for closepacked systems that are discussed in the next section.

We briefly discuss the area of application of our results. Analysis of the CCFs presented here can be applied, for example, to the results of snapshot experiments performed at free-electron laser (FEL) pulsed sources (or synchrotron sources), when the duration of the pulse (or measurement time) is much shorter than any relaxation times characteristic of the system under investigation. Most of the limiting cases discussed here will be valid for systems containing a large number of LSs. This is different from the analysis of CCFs performed in recent publications. ${ }^{19,20}$ The authors of these publications proposed to average the angular CCF [Eq. (3)] for a set of diffraction patterns measured on a large number of disordered systems, each containing a limited number of LSs in different positions and orientations. Our general formalism, developed in this paper, gives a natural explanation to the results obtained in Refs. 19 and 20. For example, in the case of a dilute system, averaging CCFs for many realizations of the system, characterized by the uniform distribution of orientations of LSs, will give a finite result that scales with the number of LSs as $\left\langle A^{2}\right\rangle=1 / N$. We will discuss the consequences of this analysis in more detail in a forthcoming publication.

\section{B. Close-packed systems}

In the case of a dense system, when the average distance $D$ between clusters is on the order of the size $d$ of a single

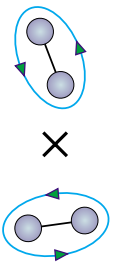

Term 1

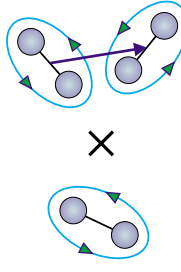

Term 2

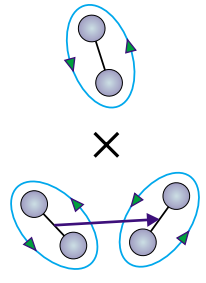

Term 3

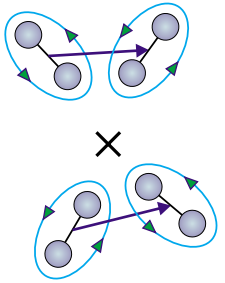

Term 4
FIG. 5. (Color online) Schematic illustration of different types of correlations contributing to the Fourier components $C_{q_{1}, q_{2}}^{n}$ of the angular CCF corresponding to four different terms in Eq. (38) (see text).

cluster, the second sum in Eq. (22) cannot be neglected. It can significantly affect the Fourier spectrum of the angular CCF. Taking both terms of Eq. (22) into account, the Fourier coefficients of the angular CCF can be written as the following sum of four terms:

$$
\begin{aligned}
& C_{q_{1}, q_{2}}^{n} \propto S_{1}^{n}+S_{2}^{n}+S_{3}^{n}+S_{4}^{n}
\end{aligned}
$$

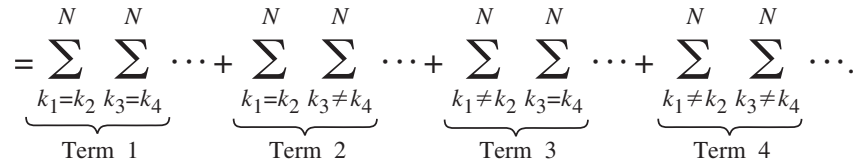

A schematic illustration of correlations corresponding to these four terms is shown in Fig. 5. The first term is a product of two two-point correlation functions, each of which correlates a separate LS with itself and, therefore, contains the information only on the internal structure of LSs. This term does not depend on the density of a disordered system and was considered in detail in the previous section. The second and the third terms are the products of two two-point correlation functions, one of which depends only on the internal structure of a LS and another one correlates different LSs, separated in space. The fourth term is a product of two two-point correlation functions each of which defines correlations of spatially separated LSs. Clearly, the terms $S_{2}^{n}, S_{3}^{n}$, and $S_{4}^{n}$ depend on the density of a disordered system. In the following, we consider in detail each term of Eq. (38).

Taking into consideration the results obtained in the previous section, the contribution of $S_{2}^{n}$ and $S_{3}^{n}$ in Eq. (38) can be written as

$$
\begin{aligned}
S_{2}^{n}+S_{3}^{n}= & N\left[\left\langle e^{i n \phi}\right\rangle_{\phi} L^{n *}\left(q_{1}\right) \sum_{k_{1} \neq k_{2}}^{N} L_{k_{1}, k_{2}}^{n}\left(q_{2}\right)\right. \\
& \left.+\left\langle e^{-i n \phi}\right\rangle_{\phi} \sum_{k_{3} \neq k_{4}}^{N} L_{k_{3}, k_{4}}^{n *}\left(q_{1}\right) L^{n}\left(q_{2}\right)\right],
\end{aligned}
$$

where $L^{n}\left(q_{j}\right)$ and $L_{k_{1}, k_{2}}^{n}\left(q_{j}\right)$ are defined in Eqs. (26) and (B1), and the angular average $\left\langle e^{i n \phi}\right\rangle_{\phi}$ is defined in Eq. (31). According to its structure the nonzero contributions of these terms are defined by the same selection rules $\left(L^{n}\left(q_{j}\right) \neq 0\right)$ as for the term $S_{1}^{n}$. In addition, the value of nonzero contribu- 


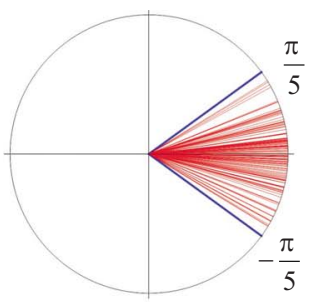

(a)
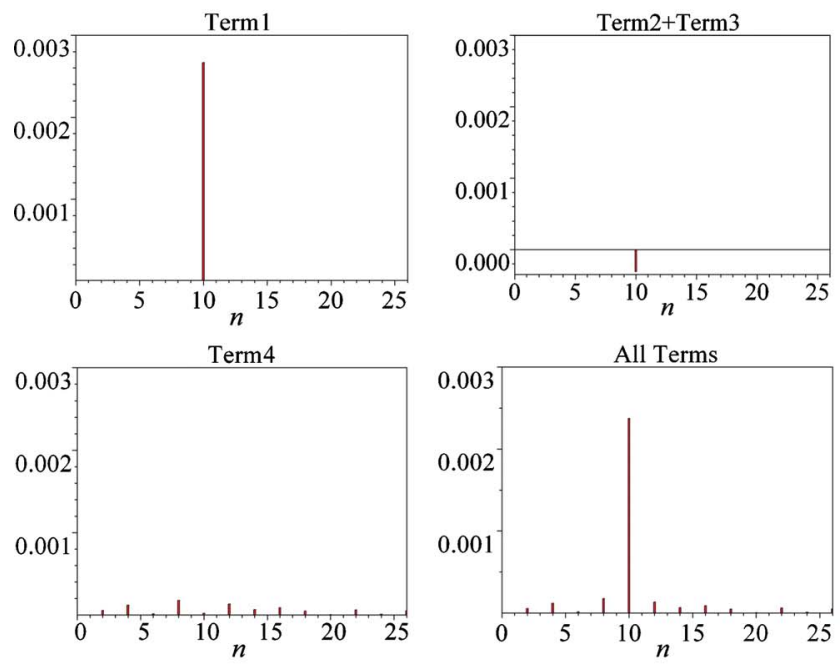

(b)
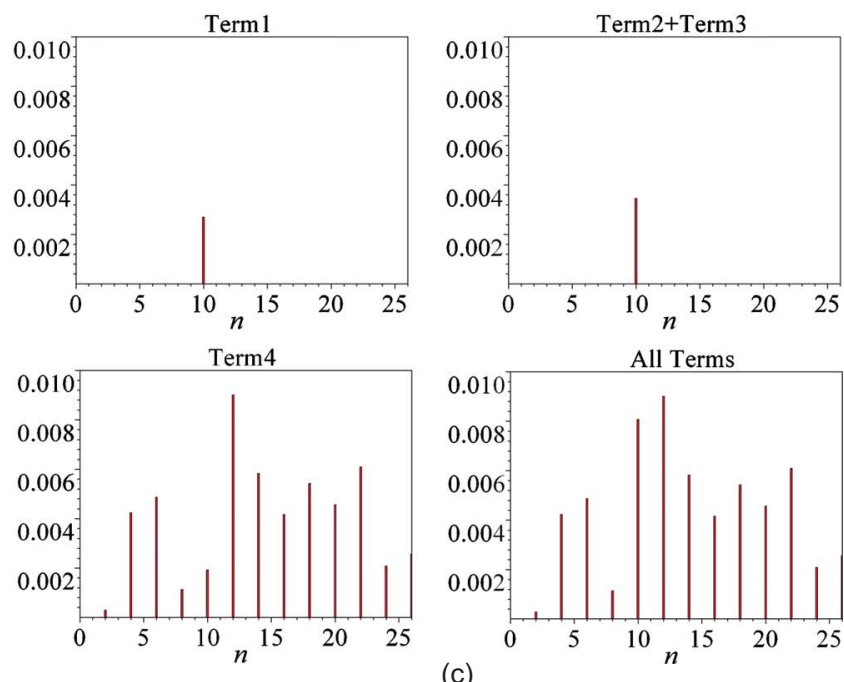

(c)

FIG. 6. (Color online) Normalized contributions of different terms to the Fourier coefficients $C_{q}^{n}$ at $q=0.037 \mathrm{~nm}^{-1}$. A 2D disordered system consisting of pentagonal clusters was considered. (a) Gaussian distribution of the in-plane angular orientations of the pentagonal clusters (with a standard deviation $\sigma=0.2 \times 2 \pi / 5$ ). Two bold lines bound a central angle $\phi=2 \pi / 5$. (b) The case of a dilute system $(D / d=150)$. (c) The case of a close-packed system $(D / d$ $=1.5)$.

tions will be modulated by the orientational order parameter $\left\langle e^{i n \phi}\right\rangle$ and the spatial correlations between different LSs defined by $L_{k_{1}, k_{2}}^{n}\left(q_{j}\right)$.

Finally, for the fourth term in Eq. (38) we have

$$
S_{4}^{n}=\sum_{k_{1} \neq k_{2}}^{N} \sum_{k_{3} \neq k_{4}}^{N} L_{k_{1}, k_{2}}^{n *}\left(q_{1}\right) L_{k_{3}, k_{4}}^{n}\left(q_{2}\right),
$$

where $L_{k_{1}, k_{2}}^{n}\left(q_{j}\right)$ are defined in Eq. (B1). This term is determined only by the spatial correlations between different LSs and can play a significant role in the close-packed systems.

We support our discussion by the calculations of the normalized contributions of all four terms in Eq. (38) to the Fourier coefficients $C_{q}^{n}$ (for the case $q_{1}=q_{2}=q$ ). In our simulations we consider a $2 \mathrm{D}$ disordered system consisting of pentagonal clusters [see Appendix $\mathrm{C}$ and Fig. 4(d)]. A highdensity system with $D / d=1.5$ and a low-density system with $D / d=150$ were considered in these calculations, where $D$ is an average distance between the clusters and $d$ is the size of a pentagonal cluster, being $d=440 \mathrm{~nm}$ in our case. Both systems contain 121 pentagonal clusters and were characterized by the same set of in-plane angular cluster orientations $\left\{\phi_{k}\right\}$. These angles were defined by a Gaussian distribution [Eq. (35)], with a standard deviation $\sigma=0.2 \times 2 \pi / 5$ [see Fig. $6(a)$. This distribution of angles covers all possible orientations for a fivefold pentagonal cluster.

The results of the calculations for $q=0.037 \mathrm{~nm}^{-1}$ are shown in Figs. 6(b) and 6(c) (here, for clarity, only the first 25 Fourier coefficients are shown). In the case of a dilute system [Fig. 6(b)], the contribution of the Term 1 strongly dominates the contributions of all other terms. It reveals fivefold symmetry by the presence of the Fourier coefficient with $n=10$. Higher orders $(n=20,30, \ldots)$ are not present due to the choice of the $q$ value. For this system the contribution from spatial correlations between different structures is negligible. In the opposite case of the close-packed system [Fig. 6(c)], the contribution from spatial correlations (Term 4) dominates the contribution from the local symmetry of individual clusters (Term 1 ). The fourth term significantly modifies the frequency spectra, in particular, by adding the coefficients which are not related to the internal structure of clusters. In this case, the Fourier coefficients with $n=10$ and $n=12$ are dominant in the shown range of the $C_{q}^{n}$ spectrum but only one of them, with $n=10$, is related to the internal structure of clusters.

Our results show that for dilute disordered systems the main contribution to the cross-correlation function $C_{q_{1}, q_{2}}(\Delta)$ is defined by the local symmetry of clusters. For a partially ordered system, one can extract this information by analyzing Fourier coefficients of the CCF. For a dense system, the spatial correlations between clusters can become dominant, and their contribution to the CCF cannot be easily separated from the contribution defined by the internal structure of clusters forming the system. Here it is important to note that in the case of partially coherent beams with the transverse coherence length smaller than the typical correlation length of the medium range order, the information on these important spatial correlations between clusters in close-packed systems can be lost. However, experiments with incoming beams that have a coherence length on the order of the size of the cluster can give access to the local symmetry of the clusters, similar to the case of dilute systems. 


\section{CORRELATIONS IN 3D SYSTEMS. EWALD SPHERE CURVATURE EFFECTS}

In our previous discussion of scattering on 2D systems, we have seen that only even Fourier coefficients of the CCF have nonzero values. Here we will show that nonzero odd Fourier coefficients can be present when scattering to high angles from 3D systems due to Ewald sphere curvature effects. In this case full expressions [Eqs. (21a) and (21b)] containing the $z$ components of the scattering vector $q_{j}^{z}$ need to be analyzed.

To simplify our discussion, we will consider here a 3D system consisting of identical 3D clusters composed of identical point scatterers. The modified electron density [Eq. (18)] of a cluster can be defined in the following form:

$$
\tilde{\rho}_{k}\left(\mathbf{r}^{\perp}, q_{j}^{z}\right)=f\left(q_{j}\right) \sum_{i=1}^{N_{s}} \delta\left(\mathbf{r}^{\perp}-\mathbf{r}_{i}^{\perp}\right) e^{-i q_{j}^{z} z_{i}}
$$

where $f\left(q_{j}\right)$ is a form factor of a scatterer and $N_{s}$ is a number of scatterers in the cluster. The coordinates $\left(\mathbf{r}_{i}^{\perp}, z_{i}\right)$ define the position of the $i$ th scatterer inside the cluster $k$. Performing the integration in Eq. (21b) gives

$$
L_{k_{1}, k_{2}}^{n}\left(q_{j}^{\perp}, q_{j}^{z}\right)=\left|f\left(q_{j}\right)\right|^{2} \sum_{l, m=1}^{N_{s}} e^{-i q_{j}^{z} z_{m l} J_{n}}\left(q_{j}^{\perp}\left|\mathbf{R}_{k_{2}, k_{1}}^{\perp m l}\right|\right) e^{-i n \phi_{\mathbf{R}_{k_{2}, k_{1}}^{\perp m l}},}
$$

where the summation over index $l$ is performed over the positions of scatterers in the cluster $k_{1}$, and the summation over index $m$ is performed over the positions of scatterers in the cluster $k_{2}$. Substituting this expression into Eq. (21a) we obtain

$$
\begin{aligned}
& I^{n}\left(q_{j}^{\perp}, q_{j}^{z}\right) \\
& =(i)^{n}\left|f\left(q_{j}\right)\right|^{2} \sum_{k_{1}, k_{2}=1}^{N} \sum_{l, m=1}^{N_{s}} e^{-i q_{j}^{z} Z_{k_{2}, k_{1}}^{m l} J_{n}}\left(q_{j}^{\perp}\left|\mathbf{R}_{k_{2}, k_{1}}^{\perp m l}\right|\right) e^{-i n \phi_{\mathbf{R}_{k_{2}, k_{1}}^{\perp m l}} .}
\end{aligned}
$$

We note here that for $n \neq 0$ the terms with $k_{1}=k_{2}$ and $l=m$ are equal to zero. Taking into account that the terms with interchanged indices, i.e., $k_{1}, k_{2}$ and $k_{2}, k_{1}$, as well as $l, m$ and $m, l$, differ from each other by a change in the sign of $Z_{k_{2}, k_{1}}^{m l}$ and by an additional factor $(-1)^{n}$, which arises due to the change in the phase $\phi_{\mathbf{R}_{k_{2}, k_{1}}^{\perp m l}}=\phi_{\mathbf{R}_{k_{1}, k_{2}}^{\perp l m}}+\pi$, we have for even values of $n$ in Eq. (43),

$$
I^{n}\left(q_{j}^{\perp}, q_{j}^{z}\right)=2(i)^{n}\left|f\left(q_{j}\right)\right|^{2} \sum_{\substack{1 \leq k_{1} \leq N \\ k_{1} \leq k_{2} \leq N}} \sum_{\substack{1 \leq l \leq N_{s} \\ l \leq m \leq N_{s}}} \cos \left(q_{j}^{z} Z_{k_{2}, k_{1}}^{m l}\right) J_{n}\left(q_{j}^{\perp} \mid \mathbf{R}_{k_{2}, k_{1}}^{\perp m l}\right) e^{-i n \phi_{\mathbf{R}_{k_{2}, k_{1}}}}
$$

and for odd values of $n$,

$$
I^{n}\left(q_{j}^{\perp}, q_{j}^{z}\right)=2(i)^{n+1}\left|f\left(q_{j}\right)\right|^{2} \sum_{\substack{1 \leq k_{1} \leq N \\ k_{1} \leq k_{2} \leq N}} \sum_{\substack{1 \leq l \leq N_{s} \\ l \leq m \leq N_{s}}} \sin \left(q_{j}^{z} Z_{k_{2}, k_{1}}^{m l}\right) J_{n}\left(q_{j}^{\perp}\left|\mathbf{R}_{k_{2}, k_{1}}^{\perp m l}\right|\right) e^{-i n \phi_{\mathbf{R}_{2}, k_{1}}^{\perp m l} .}
$$

From the performed analysis we can see that, due to the curvature of the Ewald sphere (nonzero $q_{j}^{z}$ component), we obtain nonzero odd Fourier components of the CCF when scattering from a 3D system. These components become negligibly small for experimental conditions corresponding to a flat Ewald sphere, considered in the previous section. A detailed discussion of the differences between the correlation analysis of 2D and 3D systems, based on simulations, will be given in a forthcoming paper.

\section{CONCLUSIONS AND OUTLOOK}

The basic results of this paper, Eqs. (21a) and (21b), are characterized by the following structure. (1) They break up as a sum over LS pairs. Two points belonging to two LSs of a pair define a phase factor through the angle of the projection of their connecting vector on the $(x, y)$ plane.

(2) Additional oscillating factors come from the Bessel functions of integer order depending on the projections on the $(x, y)$ plane of the connecting vector and of the scattering vector $\mathbf{q}_{j}^{\perp}$; and also from the effective density $\tilde{\rho}_{k}\left(\mathbf{r}^{\perp}, q_{j}^{z}\right)$. Note that, in the far-field diffraction limit adopted here, for scattering at small angles (small $q_{z}$ ), odd $m$ values are strongly suppressed in comparison to even ones by the trigonometric prefactors. This is in disagreement with the strong $m=5$ components observed experimentally ${ }^{1}$ and will need an additional analysis, for example, in the near-field scattering geometry.

(3) Classes of LS pairs for which the oscillating factors systematically have the same sign give the largest contribution to the sum for a given $n$. This is the case for the $k_{1}$ $=k_{2}$ pairs, and the (purely two-dimensional) examples described in Appendix $\mathrm{C}$ show how the nonvanishing values of $n$ are related to the rotational symmetry of the LSs around a common axis aligned with the direction of incidence. However, each LS contributes a value multiplied by a phase factor related to its orientation with respect to a reference direction in the plane; it is then easy to see that if the ensemble of illuminated LSs has a completely random orientation around the $n$-fold axis that the sum vanishes. In this case, indeed, 
LSs rotated by $\pi / n$ with respect to a given direction are as probable as those lined up in that direction and their respective contributions cancel in the total result. This is in agreement with the concept of bond-orientational order. ${ }^{8}$ If a nonzero Fourier coefficient is observed, it implies either a preferential alignment along a given direction, either by a specific physical reason or, alternatively, because the ensemble of probed LSs is small enough to display pronounced fluctuations from the average uniform distribution of orientations. Another interesting possibility, in view of the imminent availability of free-electron laser sources, could occur if the acquisition time is short enough to provide an "instantaneous" view, without effectively performing a time average that necessarily restores the equal probability of all orientations. This may indeed be already the case in experiments involving very slow dynamics, as may be the case in Ref. 1.

(4) In a three-dimensional fluid, the order parameter defined above is contributed to only by those molecules for which the $n$-fold axis is, at least to some degree of approximation, aligned to the direction of incidence. This probably explains why the observed Fourier components, especially in the intensity, but also in the CCF, are weak when compared to the extremely marked ones observed in hexatic liquid crystals, which are stacks of two-dimensional manifolds. ${ }^{3-5}$ The same difference in the performance of high-order CCFs was observed in the scattering of the coherent laser light on $2 \mathrm{D}$ and $3 \mathrm{D}$ colloid systems. ${ }^{6,21}$ It is tempting to speculate that the subset of LSs with an approximate lineup of a symmetry axis, in a three-dimensional system, is always "dilute," in the sense that it is constituted by a small fraction of the total number of molecules or clusters. This would allow the application of results obtained in this paper for the dilute limit also to systems which are, in the three-dimensional sense, close packed. In the companion paper, simulations are performed also with the purpose of establishing the extent of the deviation from perfect alignment of the symmetry axis which is compatible with an observable contribution to the CCF signal. It is important to bridge the gap between a twodimensional theoretical interpretation that seems to arise naturally from the experimental geometry and the threedimensional isotropy of ordinary samples.
There are various directions that future experiments may explore. It would certainly be very interesting to monitor the CCF signal in a system in which a controllable experimental parameter (e.g., temperature, an electric, or magnetic field) may provide a way to vary the degree of alignment of a symmetry axis; or in which the bond-orientational order is well characterized.

\section{ACKNOWLEDGMENTS}

We acknowledge the discussion of the results of this work with H. Dosch, G. Grübel, Ch. Gutt, and P. Wochner, useful correspondence with D. Saldin and J. Spence, and the continuing interest and support for the entire project of E. Weckert.

\section{APPENDIX A}

Here we calculate the Fourier coefficients

$$
I^{n}\left(q^{\perp}, q^{z}\right)=\frac{1}{2 \pi} \int_{0}^{2 \pi} I(\mathbf{q}) e^{-i n \varphi} d \varphi
$$

of the intensity scattered at certain momentum transfer vector $\mathbf{q}$, defined in Eq. (17). The scalar product $\mathbf{q}^{\perp} \cdot \mathbf{R}_{k_{2}, k_{1}}^{\perp 21}$ in the exponent of Eq. (17) can be written as

$$
\mathbf{q}^{\perp} \cdot \mathbf{R}_{k_{2}, k_{1}}^{\perp 21}=q^{\perp} \cdot\left|\mathbf{R}_{k_{2}, k_{1}}^{\perp 21}\right| \cos \left(\varphi-\phi_{\mathbf{R}_{k_{2}, k_{1}}^{\perp 21}}\right),
$$

where $q^{\perp}$ and $\varphi$ are the polar coordinates of the perpendicular component of the vector $\mathbf{q}^{\perp}$ [see Fig. 3(a)], and $\left|\mathbf{R}_{k_{2}, k_{1}}^{\perp 21}\right|$ and $\phi_{\mathbf{R}_{k_{2}, k_{1}}^{\perp 21}}$ are the polar coordinates of the perpendicular components of the vector $\mathbf{R}_{k_{2}, k_{1}}^{\perp 21}$ (see Fig. 2). Substituting this expression in Eq. (17) and using the Jacobi-Anger expansion $^{22}$ of the exponential functions in series of Bessel functions $J_{n}(\rho)$ of the first kind of integer order $m$,

$$
e^{i \rho \cos \varphi}=\sum_{m=-\infty}^{\infty}(i)^{m} J_{m}(\rho) e^{i m \varphi}
$$

we can write

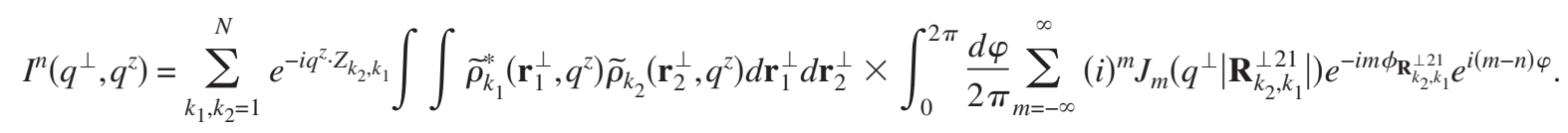

Integration over $\varphi$ in Eq. (A3) gives

$$
\int_{0}^{2 \pi}(d \varphi / 2 \pi) \exp [i(m-n) \varphi]=\delta_{m, n}
$$

where $\delta_{m, n}$ is the Kroneker symbol. Substitution of the result of this integration into Eq. (A3) finally gives

$$
I^{n}\left(q^{\perp}, q^{z}\right)=(i)^{n} \sum_{k_{1}, k_{2}=1}^{N} e^{-i q^{z} \cdot Z_{k_{2}, k_{1}}} \iint \widetilde{\rho}_{k_{1}}^{*}\left(\mathbf{r}_{1}^{\perp}, q^{z}\right) \widetilde{\rho}_{k_{2}}\left(\mathbf{r}_{2}^{\perp}, q^{z}\right) d \mathbf{r}_{1}^{\perp} d \mathbf{r}_{2}^{\perp} \times J_{n}\left(q^{\perp}\left|\mathbf{R}_{k_{2}, k_{1}}^{\perp 21}\right|\right) e^{-i n \phi_{\mathbf{R}_{k_{2}, k_{1}}^{\perp 21}} .}
$$

It is clear from the definition Eq. (A1) that 


$$
\langle I(\mathbf{q}, \varphi)\rangle_{\varphi}=I^{0}\left(q^{\perp}, q^{z}\right)=\sum_{k_{1}, k_{2}=1}^{N} e^{-i q^{z} \cdot Z_{k_{2}, k_{1}}} \iint d \mathbf{r}_{1}^{\perp} d \mathbf{r}_{2}^{\perp} \widetilde{\rho}_{k_{1}}^{*}\left(\mathbf{r}_{1}^{\perp}, q^{z}\right) \widetilde{\rho}_{k_{2}}\left(\mathbf{r}_{2}^{\perp}, q^{z}\right) J_{0}\left(q^{\perp}\left|\mathbf{R}_{k_{2}, k_{1}}^{\perp 21}\right|\right) .
$$

These results imply that the Fourier coefficients $D_{I}^{n}(q)$ of the normalized deviation, defined in Eq. (5), can be written as follows:

$$
D_{I}^{n}(q)= \begin{cases}{\left[I^{n}\left(q^{\perp}, q^{z}\right)\right] /\left[I^{0}\left(q^{\perp}, q^{z}\right)\right]} & \text { if } n \neq 0 \\ 0 & \text { if } n=0 .\end{cases}
$$

\section{APPENDIX B}

Here we prove that for dilute systems the main contribution to the Fourier coefficients of intensity $I^{n}\left(q_{j}\right)$ is given by the first term in the expansion [Eq. (22)]. In the case of a dilute disordered system, when typical distances between LSs are much larger than the size of a LS itself, i.e., $\left|\mathbf{R}_{k_{l}, k_{m}}^{i j}\right| \gg\left|\mathbf{r}_{i j}\right|$, we can use the following approximation for the values of the Bessel functions in the integrals $L_{k_{1}, k_{2}}^{n}\left(q_{j}, q_{j}^{z}\right)$ in Eq. $\quad(21 \mathrm{~b}): \quad J_{n}\left(q_{j}\left|\mathbf{R}_{k_{2}, k_{1}}^{21}\right|\right)=J_{n}\left(q_{j}\left|\mathbf{R}_{k_{2}, k_{1}}+\mathbf{r}_{21}\right|\right) \simeq J_{n}\left(q_{j}\left|\mathbf{R}_{k_{2}, k_{1}}\right|\right)$. Furthermore, for large values of the argument of the Bessel function $J_{n}(\rho), \rho \gg\left(n^{2} / 2-1 / 8\right)$, one can use the asymptotic expansion ${ }^{23} J_{n}(\rho) \simeq \sqrt{\frac{2}{\pi \rho}} \cos \left(\rho-\frac{n \pi}{2}-\frac{\pi}{4}\right)$. Taking all of this into account we finally get, for the integral $L_{k_{1} \neq k_{2}}^{n}$ in Eq. (21b) in the case of a $2 \mathrm{D}$ system,

$$
\begin{aligned}
& L_{k_{1} \neq k_{2}}^{n}\left(q_{j}\right)=\iint d \mathbf{r}_{1} d \mathbf{r}_{2} \widetilde{\rho}_{k_{1}}^{*}\left(\mathbf{r}_{1}\right) \widetilde{\rho}_{k_{2}}\left(\mathbf{r}_{2}\right) J_{n}\left(q_{j}\left|\mathbf{R}_{k_{2}, k_{1}}^{21}\right|\right) e^{-i n \phi_{\mathbf{R}_{k_{2}, k_{1}}^{21}},} \\
& \simeq \sqrt{\frac{2}{\pi q_{j}\left|\mathbf{R}_{k_{2}, k_{1}}\right|}} \cos \left(q_{j}\left|\mathbf{R}_{k_{2}, k_{1}}\right|-\frac{n \pi}{2}-\frac{\pi}{4}\right) e^{i n \phi_{\mathbf{R}_{k_{2}, k_{1}}} P_{k_{1}}^{*} P_{k_{2}},}
\end{aligned}
$$

where

$$
P_{k}=\int d \mathbf{r} \widetilde{\rho}_{k}(\mathbf{r}) .
$$

In deriving Eq. (B2) we also used an approximation $\phi_{\mathbf{R}_{k_{2}, k_{1}}^{\perp 21}}$ $\approx \phi_{\mathbf{R}_{k_{2}, k_{1}}}$ that is valid for dilute systems and means that this phase does not depend on individual orientations of LSs but is determined by their positions in the system.
We analyze now this asymptotic behavior of the second sum in Eq. (22). According to Eq. (B2), for the given $q_{j}$ value the function $L_{k_{1} \neq k_{2}}^{n}\left(q_{j}\right)$ decays as $1 / \sqrt{q_{j}\left|\mathbf{R}_{k_{2}, k_{1}}\right|}$ with the increase in the distance $\mathbf{R}_{k_{2}, k_{1}}$ between the LSs. At the same time, it can be noted that the sum $\Sigma_{k_{1} \neq k_{2}}^{N} \cdots$ in Eq. (22) contains $N(N-1)$ terms comparing to $N$ terms in the sum $\sum_{k_{1}=k_{2}=k}^{N} \cdots$. However, the presence in Eq. (B2) of the exponential factor $e^{i n \phi_{\mathbf{R}_{2}, k_{1}}}$ with random phases $\phi_{\mathbf{R}_{k_{2}, k_{1}}}$, corresponding to a large number of LSs present in dilute system, will additionally reduce the contribution of the second sum in Eq. (22). Therefore, for dilute systems, the dominant contribution to the Fourier coefficients $C_{q_{1}, q_{2}}^{n}$ will be defined by the first sum in Eq. (22) with $k_{1}=k_{2}$.

\section{APPENDIX C}

We consider here simple 2D structures (clusters) with distinct rotational $m$-fold symmetries shown in Fig. 4 . We define the electron density of a cluster as a real-valued quantity in the following form:

$$
\rho\left(\mathbf{r}_{l}\right)=\sum_{i=1}^{N_{s}} f_{i}\left(q_{j}\right) \delta\left(\mathbf{r}-\mathbf{r}_{i}\right)=\sum_{i=1}^{N_{s}} f_{i}\left(q_{j}\right) \delta\left(r_{l}-r_{i}\right) \delta\left(\phi_{l}-\phi_{i}\right),
$$

where $N_{s}$ is a number of scatterers in the cluster, $f_{i}\left(q_{j}\right)$ is a scattering factor of the $i$ th scatterer in the cluster, $\mathbf{r}=(r, \phi)$, and $r$ and $\phi$ are the polar coordinates of a scatterer in the cluster. Using the definition [Eq. (C1)] and performing the integration in Eq. (26) we get

$$
L^{n}\left(q_{j}\right)=\sum_{s, t=1}^{N_{s}} f_{s}^{*}\left(q_{j}\right) f_{t}\left(q_{j}\right) J_{n}\left(q_{j}\left|\mathbf{r}_{t s}\right|\right) e^{-i n \phi_{\mathbf{r}_{s s}}}
$$

For an arbitrary cluster with $m$-fold rotational symmetry shown in Fig. 4 the following assumptions are valid: $r_{i}=a$, i.e., all scatterers in the cluster are located on equal distances from its center, $\phi_{i}=2 \pi / m \cdot(i-1), i=1, \ldots, N_{s}$, where $m$ $=N_{s}$ is a highest order of rotational symmetry in the cluster, and we also assume $f_{i}\left(q_{j}\right)=f\left(q_{j}\right)$. Using these assumptions in Eq. (C2), we derive the expressions of $L^{n}\left(q_{j}\right)$ for each of the clusters shown in Fig. 4.

(1) $m=2$, Fig. 4(a),

$$
L^{n}\left(q_{j}\right)= \begin{cases}2\left|f\left(q_{j}\right)\right|^{2}\left[J_{n}(0)+J_{n}\left(2 a q_{j}\right)\right] & \text { if } n=0 \text { and } n \bmod 2=0 \\ 0 & \text { if } n \bmod 2 \neq 0 .\end{cases}
$$

(2) $m=3$, Fig. 4(b), 


$$
L^{n}\left(q_{j}\right)= \begin{cases}3\left|f\left(q_{j}\right)\right|^{2}\left[J_{n}(0)+2 J_{n}\left(\sqrt{3} a q_{j}\right)\right] & \text { if } n=0 \text { and } n \bmod 12=0 \\ -6\left|f\left(q_{j}\right)\right|^{2} J_{n}\left(\sqrt{3} a q_{j}\right) & \text { if } n \bmod 6=0, n \bmod 12 \neq 0 \\ 0 & \text { other } n .\end{cases}
$$

(3) $m=4$, Fig. 4(c),

$$
L^{n}\left(q_{j}\right)= \begin{cases}4\left|f\left(q_{j}\right)\right|^{2}\left\{J_{n}(0)+J_{n}\left(2 a q_{j}\right)+2 J_{n}\left(\sqrt{2} a q_{j}\right)\right\} & \text { if } n=0 \text { and } n \bmod 8=0 \\ 4\left|f\left(q_{j}\right)\right|^{2}\left[J_{n}\left(2 a q_{j}\right)-2 J_{n}\left(\sqrt{2} a q_{j}\right)\right] & \text { if } n \bmod 4=0, n \bmod 8 \neq 0 \\ 0 & \text { other } n .\end{cases}
$$

(4) $m=5$, Fig. 4(d),

$$
L^{n}\left(q_{j}\right)= \begin{cases}5\left|f\left(q_{j}\right)\right|^{2}\left\{J_{n}(0)+2\left[J_{n}\left(A_{1} a q_{j}\right)+J_{n}\left(A_{2} a q_{j}\right)\right]\right\} & \text { if } n=0 \text { and } n \bmod 20=0 \\ -10\left|f\left(q_{j}\right)\right|^{2}\left[J_{n}\left(A_{1} a q_{j}\right)+J_{n}\left(A_{2} a q_{j}\right)\right] & \text { if } n \bmod 10=0, n \bmod 20 \neq 0 \\ 0 & \text { other } n .\end{cases}
$$

where $A_{1}=\sqrt{\frac{1}{2}(5-\sqrt{5})}$ and $A_{2}=\sqrt{\frac{1}{2}(5+\sqrt{5})}$.

(5) $m=6$, Fig. 4(e),

$$
L^{n}\left(q_{j}\right)= \begin{cases}6\left|f\left(q_{j}\right)\right|^{2}\left\{J_{n}(0)+2 J_{n}\left(a q_{j}\right)+J_{n}\left(2 a q_{j}\right)+2 J_{n}\left(\sqrt{3} a q_{j}\right)\right\} & \text { if } n=0 \text { and } \mathrm{n} \bmod 12=0 \\ 6\left|f\left(q_{j}\right)\right|^{2}\left[2 J_{n}\left(a q_{j}\right)+J_{n}\left(2 a q_{j}\right)-2 J_{n}\left(\sqrt{3} a q_{j}\right)\right] & \text { if } n \bmod 6=0, n \bmod 12 \neq 0 \\ 0 & \text { other } n .\end{cases}
$$

Equations (C3)-(C7) define the selection rules which determine the contributions to the $n$th coefficient $C_{q_{1}, q_{2}}^{n}$ related only to the internal structure of clusters. For instance, Eq. (C4) means that the contribution from the internal structure of the cluster shown in Fig. 4(b) to the Fourier coefficients with $n=6,18,30, \ldots$ is defined by the function $L^{n}\left(q_{j}\right)=-6\left|f\left(q_{j}\right)\right|^{2} J_{n}\left(\sqrt{3} a q_{j}\right)$; for the coefficients with $n=12,24,48, \ldots, \quad L^{n}\left(q_{j}\right)$ $=6\left|f\left(q_{j}\right)\right|^{2} J_{n}\left(\sqrt{3} a q_{j}\right)$; for $n=0$ coefficient $L^{n}\left(q_{j}\right)=3\left|f\left(q_{j}\right)\right|^{2}[1$ $\left.+2 J_{0}\left(\sqrt{3} a q_{j}\right)\right]$ while other Fourier coefficients do not contain any information on the internal structure of this particular cluster.
*Corresponding author; ivan.vartaniants@desy.de

${ }^{1}$ P. Wochner, C. Gutt, T. Autenrieth, T. Demmer, V. Bugaev, A. Diaz-Ortiz, A. Duri, F. Zontone, G. Grübel, and H. Dosch, Proc. Natl. Acad. Sci. U.S.A. 106, 11511 (2009).

${ }^{2}$ It is to be noted that the trivial angular dependence due to the linear polarization of the incoming synchrotron beam, important at large scattering angles, must be removed from the intensity.

${ }^{3}$ R. Pindak, D. E. Moncton, S. C. Davey, and J. W. Goodby, Phys. Rev. Lett. 46, 1135 (1981).

${ }^{4}$ E. Górecka, L. Chen, W. Pyżuk, A. Krówczyński, and S. Kumar, Phys. Rev. E 50, 2863 (1994).

${ }^{5}$ C. F. Chou, A. J. Jin, S. W. Hui, C. C. Huang, and J. T. Ho, Science 280, 1424 (1998).

${ }^{6}$ N. A. Clark, B. J. Ackerson, and A. J. Hurd, Phys. Rev. Lett. 50, 1459 (1983)

${ }^{7}$ P. M. Chaikin and T. C. Lubensky, Principles of Condensed Matter Physics (Cambridge University Press, Cambridge, England, 1995).

${ }^{8}$ R. Bruinsma and D. R. Nelson, Phys. Rev. B 23, 402 (1981).
${ }^{9}$ Similar cross-correlation function, which includes an additional step of averaging over diffraction patterns, was introduced by Saldin et al. (Ref. 24).

${ }^{10}$ Here we used the fact that all functions in the integrand are periodic functions with the period $2 \pi$.

${ }^{11}$ In Eq. (11) we have also tacitly assumed an infinite illumination region that also means an infinitely small speckle size. In practice, due to finite-size effects (a finite size of the coherent beam, a finite size of the coherent area in the partially coherent beam, or a finite size of a sample) the size of a speckle is finite and is of the order $\Delta q \sim 1 / d$, where $d$ is a typical length at the sample position. However, these finite-size effects will not influence our further general treatment of the CCFs.

${ }^{12}$ In this section, we omit the superscript $\perp$, assuming that all vectors are defined in 2D plane.

${ }^{13}$ J. Goodman, Statistical Optics (Wiley, New York, 2000).

${ }^{14} \mathrm{~J}$. Goodman, Speckle Phenomena in Optics: Theory and Applications (Roberts and Company, Englewood, Colorado, 2007).

${ }^{15}$ Note different from Refs. 13 and 14 scaling factor $1 / N$ in the random phasor sum introduced in Eq. (31). 
${ }^{16}$ The same result for the infinite number of phases $\phi_{k}$ can be obtained just by assuming that all orientations are uniformly distributed in 2D plane, i.e., $p(\phi)=1 /(2 \pi)$. Performing the angular average $\left\langle e^{i n \phi}\right\rangle_{\phi}=1 /(2 \pi) \int e^{i n \phi} d \phi=\delta_{n, 0}$, we obtain a zero value for the orientational order parameter for all Fourier coefficients, except for the one with $n=0$, that is not contributing to the CCF [see Eqs. (9) and (10a)].

${ }^{17}$ The same result in the limit $N \rightarrow \infty$ can be obtained by direct integration [Eq. (33)] with the Gaussian probability distribution function [Eq. (35)].

${ }^{18}$ I. Vartanyants and I. Robinson, J. Synchrotron Radiat. 10, 409 (2003).
${ }^{19}$ D. K. Saldin, H. Poon, V. Shneerson, M. Howells, H. Chapman, R. A. Kirian, K. E. Schmidt, and J. C. H. Spence, Phys. Rev. B 81, 174105 (2010).

${ }^{20}$ D. K. Saldin et al., New J. Phys. 12, 035014 (2010).

${ }^{21}$ P. Pusey and J. Rarity, J. Phys. (Paris) 46, 43 (1985).

${ }^{22}$ A. Cuyt, V. B. Petersen, B. Verdonk, H. Waadeland, and W. B. Jones, Handbook of Continued Fractions for Special Functions (Springer, New York, 2008).

${ }^{23}$ M. Abramowitz and I. Stegun, Handbook of Mathematical Functions (Courier Dover, New York, 1972).

${ }^{24}$ D. K. Saldin, V. L. Shneerson, R. Fung, and A. Ourmazd, J. Phys.: Condens. Matter 21, 134014 (2009). 